\title{
Multidimensional denoising of rolling element bearings with compound fault based on tensor factorization
}

\author{
Chaofan Hu ${ }^{1}$, Yanxue Wang ${ }^{2}$ \\ 1 School of Mechanical and Electrical Engineering, Guilin University of Electronic Technology, Guilin \\ 541004, PR. China. huchaofan199215@126.com \\ 2 School of Mechanical-Electronic and Vehicle Engineering, Beijing University of Civil Engineering and \\ Architecture, Beijing 100044, PR. China. wyx1999140@126.com
}

Keywords:Tensor factorization; fault diagnosis; high order singular value decomposition; denoising

\begin{abstract}
This article presents a multidimensional denoising technique of rolling element bearings based on tensor factorization which can model a signal in the high dimensional space so as to solve multi-channel signal filtering. The vibration signal is formulated as a 4-way tensor, temporal signal, frequency and 2 data channels. Tensor model is then factorized via truncated high order singular value decomposition. L-curve criterion is adopted to find the truncation parameters used in tensor factorization. The proposed approach is applied to remove noise of bearing vibration signal on test-rigs. Experimental results showed that the proposed method can well remove noise and keep fine structures of the signal as much as possible. This tensor based multidimensional signal filtering will broaden view for dealing with heterogeneous and multiaspect data in an age of big data.
\end{abstract}

\section{Introduction}

Rolling element bearings are among the most critical components in a rotating machine. However, those key components will inevitably suffer from various faults because of harsh working conditions such as heavy load and high speed. Those failures may lead to costly downtime and catastrophic accidents. Thus, efficient and robust vibration-based fault detection techniques have been attracted considerable research over the last few decades in order to maintain a high degree of availability, reliability and operational safety [1-2].

However, measured vibration signals are generally complex and non-stationary in nature, and meanwhile fault signatures of key components are usually immersed in stochastic noise [3, 4]. As a result, it is always a challenge to develop versatile signal processing techniques that can detect crucial fault information buried in signals [5]. dual-tree complex wavelet [1] have been used for vibration signal processing. Those techniques need to select basic function and decomposition level, which partly restricts their practical applications. Nonlocal mean (NLM) has been successfully used for signal denoising in rotating machine in [6], but it is still unsuitable for signal with large noise variance [7]. Variational mode decomposition (VMD) [8] was recently applied to detect rubbing-caused features in rotary machinery. VMD and majoriation-minization based total variation denoising (TV-MM) [3] has been also developed to remove stochastic noise in raw signal and to enhance the corresponding characteristics. However, most of these techniques severely destroy the structure of original signal during removing noise and enhancing signatures, which go against for the following analysis if the denoised signal should be further used. In addition, it is difficult for most of traditional signal processing techniques to deal with heterogeneous, multiaspect data simultaneously. 
With the rapid development of multidimensional data, it is inevitable to deal with large amounts of data in high dimensional space. Tensor and tensor decomposition are very powerful and versatile tools that can model a wide variety of heterogeneous, multiaspect data. Thus, tensor decomposition, which extract useful latent information out of multiaspect data tensors, have witnessed increasing popularity and adoption. Tensors are the high order generalization of vectors and matrices and provide a useful representation of the existing real data that has the natural multi-dimensional structure [8,9].

Tensor decomposition has become surprisingly attractive for signal processing [10]. A key problem in tensor analysis tasks is to find a suitable decomposition of the observed tensor when noise exist [11], and so tensor completion/recovery is important. HOSVD is extension of matrix SVD has been proved, especially for second-order tensor (matrix), both is equivalent. Therefore, it's easy to see that fault diagnosis based on truncated HOSVD is the same as the defect based on tensor decomposition with truncated SVD. Considering n-mode singular vectors corresponding to the larger n-mode singular value in tensor decomposition capture more energy, tensor decomposition with truncated HOSVD is introduced and is applied to signal denoising and fault diagnosis.

This paper attempts to construct a forth order tensor model with 2 data channels, time, and frequency in fault diagnosis of rolling element bearings aiming at analysis of multidimensional data. Tensor decomposition is very suitable for processing multidimensional data, which is also first adopted to signal denoising and fault diagnosis of rotating machine in this work. Moreover, the L-curve method is proposed to find the regularization parameters. The remaining part of the paper is organized as follows: Section 2 introduces background theory of the proposed method briefly. The proposed fault diagnosis method described in Section 3. The proposed approach is then applied to remove noise of bearing vibration signal in Section 4. Concluding remarks are given in Section 5.

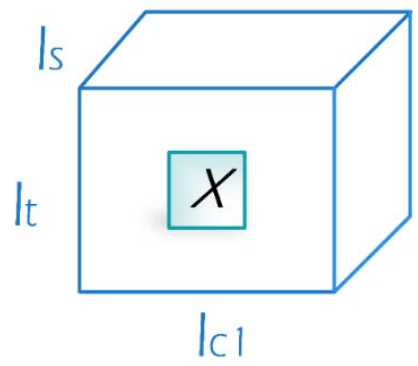

Figure 1. Third order tensor model.

\section{The basic theory of tensor factorization}

Multiway arrays, also referred to as tensors, are higher-order generalizations of vectors and matrices. A brief overview of tensor decomposition and its application can be found in [12]. A higher-order array is represented as $\mathrm{X} \in \mathrm{R}^{\mathrm{I}_{1} \times \mathrm{I}_{2} \times \ldots \mathrm{I}_{\mathrm{N}}}$, where the order of $\mathrm{X}$ is $\mathrm{N}$. Each dimension of a multiway array is called a mode. The mode- $n$ unfolding (also called matricization or flattening) of a tensor $\mathrm{X} \in$ $\mathrm{R}^{\mathrm{I}_{1} \times \mathrm{I}_{2} \times \ldots \mathrm{I}_{N}}$ is defined as unfolding $(X, n)=X(n)$, where the tensor element $\left(\mathrm{i}_{1}, \mathrm{i}_{2}, \ldots \mathrm{i}_{n}\right)$ is mapped to the matrix element $\left(i_{n}, j\right)$, which can be written below

$$
\mathrm{j}=1+\sum_{\substack{\mathrm{k}=1 \\ \mathrm{k} \neq \mathrm{n}}}^{\mathrm{N}}\left(\mathrm{i}_{\mathrm{k}}-1\right) \mathrm{J}_{\mathrm{k}} \text { with } \mathrm{J}_{\mathrm{k}}=\prod_{\substack{\mathrm{m}=1 \\ \mathrm{~m} \neq \mathrm{n}}}^{\mathrm{k}-1} \mathrm{I}_{\mathrm{m}}
$$

Therefore, $\mathrm{X}_{(\mathrm{n})} \in \mathrm{R}^{\mathrm{I}_{\mathrm{N}} \times \mathrm{J}}$ where $\mathrm{J}_{\mathrm{K}}=\prod_{\mathrm{k}=1, \mathrm{k} \neq \mathrm{n}}^{\mathrm{k}-1} \mathrm{I}_{\mathrm{k}}$. The $\mathrm{n}$-rank of an $\mathrm{N}$-dimensional tensor $\mathrm{X} \in$ $\mathrm{R}^{\mathrm{I}_{1} \times \mathrm{I}_{2} \times \ldots \mathrm{I}_{\mathrm{N}}}$, denoted by $\mathrm{R}(\mathrm{n})$, is the rank of the mode-n unfolding matrix $\mathrm{X}(\mathrm{n})$. The inner product of two 
same-size tensors $A, B \in R^{I_{1} \times I_{2} \times \ldots I_{N}}$ is defined as the sum of the products of their entries, i.e.

$$
(A, B)=\sum_{i_{1}} \sum_{i_{2}} \ldots \ldots \sum_{i_{n}} a_{i_{1} \ldots i_{k} \ldots i_{n}} b_{i_{1} \ldots i_{k} \ldots i_{n}} \text {. }
$$

The corresponding Frobenius norm is $\|\mathrm{X}\|_{\mathrm{F}}=\sqrt{(\mathrm{X}, \mathrm{X})}$. For any $1 \leq \mathrm{n} \leq \mathrm{N}$, the $\mathrm{n}$-mode (matrix) product of a tensor $A \in R^{I_{1} \times I_{2} \times \ldots I_{N}}$ with a matrix $M \in R^{I_{N} \times J}$ is denoted by $A \times{ }_{n} M$. In terms of flattened matrix, the n-mode product can be expressed as,

$$
\mathrm{y}=\mathrm{A} \times_{\mathrm{n}} \mathrm{M} \Leftrightarrow \mathrm{Y}_{(\mathrm{n})}=\mathrm{MA}_{(\mathrm{n})}
$$

Tucker models developed in [12], [13] and [14] are a common way to express a tensor factorization. Tucker decomposition naturally generalizes orthonormal subspaces corresponding to the left/right singular matrix computed by the matrix SVD [15]. A tensor $\mathrm{X} \in \mathrm{R}^{\mathrm{I}_{1} \times \mathrm{I}_{2} \times \ldots \mathrm{I}_{\mathrm{N}}}$ can be written via the Tucker model,

$$
X=G \times{ }_{1} A_{1} \times A_{2} \ldots \times_{n} A_{n}
$$

where $A_{i} \in R^{I_{i} \times R_{i}}$ contains basis vectors, $G \in R^{R_{1} \times R_{2} \ldots \times R_{N}}$ is called the core tensor and its entries show the level of interaction between the different components. Tucker decomposition $(\mathrm{N}=3)$ is illustrated in Fig.1. Tucker decomposition $(\mathrm{N}=4)$ is based on Tucker decomposition $(\mathrm{N}=3)$. Forth order tensor model is established by third order tensor model with adding a dimension. The tensor is decomposed as a linear combination of basis vectors in different modes.

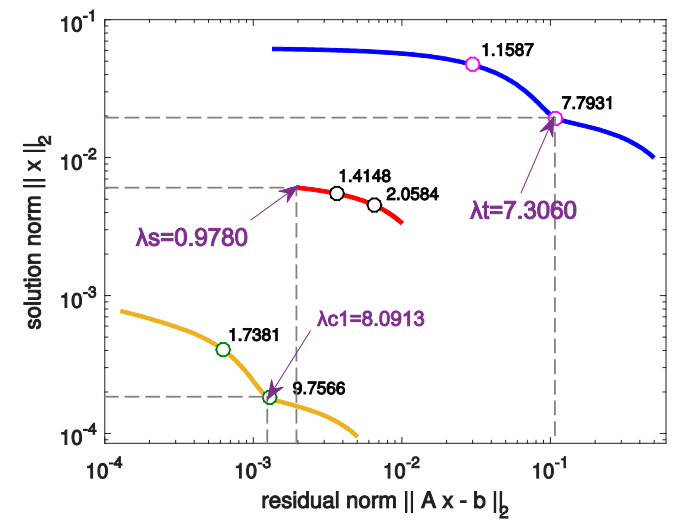

Figure 2. The truncation parameters for compound fault

\section{Multidimensional denoising with tensor factorization}

Signal processing method based on tensor factorization develops multi-way filtering, which is also very helpful for the analysis of big data. Suppose that the multidimensional signal is represented by a tensor $\mathrm{T}$, the signal $\mathrm{R}$ with the additional noise $\mathrm{N}$ is given by

$$
\mathrm{R}=\mathrm{T}+\mathrm{N}
$$

in which $\mathrm{R}, \mathrm{T}, \mathrm{N} \in \mathrm{R}^{\mathrm{I}_{1} \times \mathrm{I}_{2} \ldots \times \mathrm{I}_{\mathrm{N}}}$ are all $\mathrm{N}$-order tensor. Therefore, $\mathrm{T}$ and $\mathrm{N}$ separately represent the clean signal and the noise in this model. The estimated noiseless signal using multidimensional filtering can be thus written below [16-18].

$$
\widehat{\mathrm{T}}=\mathrm{R} \times{ }_{1} \mathrm{H}^{(1)} \times_{2} \mathrm{H}^{(2)} \times_{3} \ldots \times_{\mathrm{N}} \mathrm{H}^{(\mathrm{N})}
$$

where $\mathrm{H}^{(\mathrm{N})}$ is denoted as a n-mode filter. Suppose that the interference noise $\mathrm{N}$ is independent of the target signal $\mathrm{T}$, and the $\mathrm{n}$-modular rank $\mathrm{K}_{\mathrm{n}}$ of the tensor $\mathrm{R}$ is less than the dimension $\mathrm{I}_{\mathrm{n}}$ of its $\mathrm{n}$ mode ( $\mathrm{K}_{\mathrm{n}}<\mathrm{I}_{\mathrm{n}}, \forall \mathrm{n}=1: \mathrm{N}$ ). The tensor space can be divided into two orthogonal vector spaces which are the signal subspace and the noise subspace, defined as $E_{1}$ and $E_{2}$, respectively. $E_{1}$ is a subspace of dimension $K_{n}$, which is obtained by singular vectors corresponding to the maximum $K_{n}$ singular values of $T_{n}$. $E_{2}$ is a subspace of dimension $I_{n}-K_{n}$, which is obtained by singular vectors corresponding to the maximum $I_{n}-K_{n}$ singular values of $T_{n}$. 
Since a tensor is a multidimensional array, specially, an N-way or Nth-order tensor is an element of the tensor product of $\mathrm{N}$ vector spaces [19]. Each tensor has its own coordinate system. Third -order tensor noisy signal $\mathrm{X}$ can be represented by the formula

$$
\mathrm{X}=\mathrm{G} \times{ }_{1} \mathrm{P}^{(1)} \times_{2} \mathrm{P}^{(2)} \times{ }_{3} \mathrm{P}^{(3)} \times_{4} \mathrm{P}^{(4)}
$$

where $P^{(j)} \in R^{I_{j} \times R_{j}}\left(j=1,2,3,4 R_{j} \ll I_{j}\right)$ is a factor matrix which represents the important components of each module, and $\mathrm{G} \in \mathrm{R}^{\mathrm{R}_{1} \times \mathrm{R}_{2} \times \mathrm{R}_{3} \times \mathrm{R}_{4}}$ is called the core tensor.

In order to reduce the dimension of the signal tensor, the key is to select the proper left singular vector in the factor matrix $\mathrm{P}^{(\mathrm{j})}$, so that the target signal can be best preserved, and the signal distortion is not caused. Assume that the number of vectors to be retained in each factor matrix is known to be $\lambda_{\mathrm{t}}, \lambda_{\mathrm{c} 1}, \lambda_{\mathrm{c} 2}, \lambda_{\mathrm{s}}$ and the matrices are $\mathrm{U}^{(1)}, \mathrm{U}^{(2)}, \mathrm{U}^{(3)}, \mathrm{U}^{(4)}$, thus, a new core tensor can be obtained using

$$
\widetilde{G}=X \times_{1} U^{(1)^{T}} \times_{2} U^{(2)^{T}} \times_{3} U^{(3)^{T}} \times_{4} U^{(4)^{T}}
$$

Then the purified signal tensor can be restored by the following reconstruction

$$
\widetilde{S}=\widetilde{G} \times_{1} U^{(1)} \times_{2} U^{(2)} \times_{3} U^{(3)} \times_{4} U^{(4)}
$$

Finally, the new channel data is obtained by inverse transformation according to the original tensor construction.

The partial dimension of the data in the tensor high-dimensional space is composed of noisy signals, the target pure signal tensor is thus actually made up of lower dimensions. This allows us to remove the noise redundancy by tensor decomposition. The truncated HOSVD is a popular method for analysis tensor decomposition. The method requires the choice of a truncation index, which affects the quality of the computed approximate solution. When the truncated HOSVD is processed, the truncation parameters are obtained according to the L-curve criterion. Truncation parameters $\lambda_{t}, \lambda_{c 1}, \lambda_{c 2}, \lambda_{s}$ are separately round to an integer. Then the vibration signal is modeled a tensor $\mathrm{X} \in \mathrm{R}^{\lambda_{\mathrm{t}} \times \lambda_{\mathrm{c} 1} \times \lambda_{\mathrm{c} 2} \times \lambda_{\mathrm{s}}}$

\section{Bearing vibration signal denoising}

In this section, a kind of bearing (inner race defect) signals acquired on MFS-Magnum test-rig are adopted to verify the effectiveness of the proposed method. Type of ER-12K bearings are used in experiments, whose specifications are provided in Table 1.

Table 1

\begin{tabular}{|c|c|c|c|c|}
\hline \multicolumn{7}{|c|}{ Specifications of the bearing } \\
\hline $\begin{array}{c}\text { Inside } \\
\text { diameter } \\
(\mathbf{m m})\end{array}$ & $\begin{array}{c}\text { Outside } \\
\text { diameter } \\
\mathbf{( m m )}\end{array}$ & $\begin{array}{c}\text { Pitch } \\
\text { diameter } \\
\mathbf{( m m})\end{array}$ & $\begin{array}{c}\text { Number of } \\
\text { rolling } \\
\text { elements(mm) }\end{array}$ & $\begin{array}{c}\text { Ball } \\
\text { diameter } \\
\text { (mm) }\end{array}$ \\
\hline 25.4 & 52 & 33.4772 & 8 & 7.9375 \\
\hline
\end{tabular}

\subsection{Bearing compound fault detection}

As is well known, vibration signal should present the impulsive features due to the presence of defect in the rolling bearing. However, weak signatures in the time domain are often contaminated because of the existing strong ambient noise.

The rotating frequency of the input shaft is set to $29.85 \mathrm{~Hz}$ in this experiment. The vibration signal is modeled a forth tensor $\mathrm{X} \in \mathrm{R}^{\mathrm{I}_{\mathrm{t}} \times \mathrm{I}_{\mathrm{c} 1} \times \mathrm{I}_{\mathrm{c} 2} \times \mathrm{I}_{\mathrm{s}}}$, which according to 2 channels, time and frequency. where $\mathrm{I}_{t}$ represents time, $I_{c}$ represents data channel, $I_{s}$ represents frequency.

When the tensor decomposition is processed, the truncation parameter is obtained according to the $\mathrm{L}$ curve criterion in Fig. 2. Truncation parameters are rounded with their integer, four parameters are set as $\tilde{\lambda}_{\mathrm{t}}=7, \tilde{\lambda}_{\mathrm{c} 1}=8, \tilde{\lambda}_{\mathrm{c} 2}=0$ and $\tilde{\lambda}_{\mathrm{s}}=1$. Then this vibration signal is modeled a tensor $\mathrm{X} \in \mathrm{R}^{7 \times 8 \times 0 \times 1}$ after tensor 
decomposition.

The raw vibration signal of bearing with compound fault defect is shown in Fig. 3(a). The purified signal using tensor decomposition is shown in Fig. 3(b).
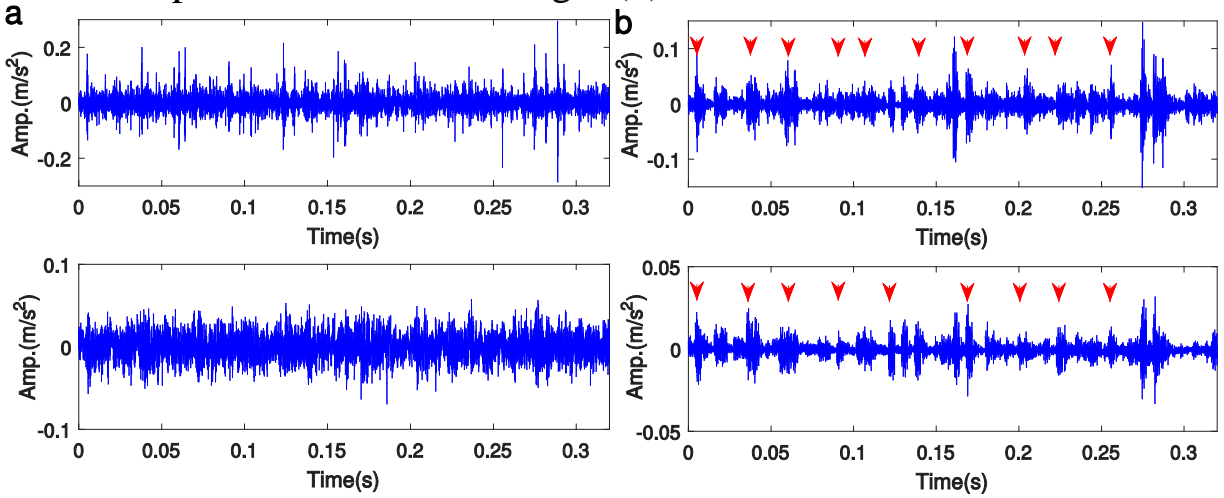

Figure 3. The vibration signal of compound fault: (a) Original vibration signal (b) the filtered signal with tensor decomposition

\section{Conclusions}

A novel multidimensional noise reduction technique of rotating machine is presented in this work

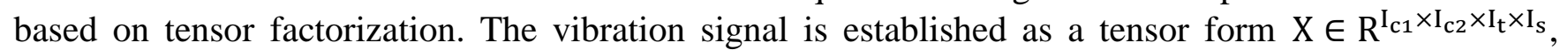
denoising problem is thus solved through tensor tool in high dimensional space. Tucker decomposition is applied to filter each module of the tensor so that the noisy signal tensor can be purified and ideal noise component is achieved. L-curve criterion is adopted to find the truncation parameters used in tensor factorization, which guarantees regularized solution be a good approximation of the exact solution in denoising. The effectiveness of the proposed new method was verified for detecting compound fault of bearing. Our future work will consider further optimization which how to optimize formation of tensors on the basis of this study to further improve the proposed method.

Moreover, the power of tensor decomposition in processing heterogeneous, multiaspect data is not brought into full play, which will be investigated in the future work. Now exploring the signal processing method based on tensor analysis will provide a broad stage for the application of multidimensional data processing in an age of big data.

\section{Acknowledgment}

The financial sponsorship from the project of National Natural Science Foundation of China (51475098, 61463010 and 51405093) and Guangxi Natural Science Foundation (2016GXNSFFA380008) are gratefully acknowledged. It is also sponsored by Guangxi key laboratory of manufacturing system \& advanced manufacturing technology (15-140-30-001Z, 16-380-12-004Z, 16-380-12-011K, and 14-04515-006Z), Innovation Project of Guangxi Graduate Education (YCSW2017136) and basic ability promotion project for young and middle-aged teachers in Guangxi Province (Grant No. 2017KY0207).

\section{References}

[1] Wang Y, He Z, Zi Y. Enhancement of signal denoising and multiple fault signatures detecting in rotating machinery using dual-tree complex wavelet transform[J]. Mech. Syst. Signal Process. , 2010, 24(1): 119-137.

[2] Wang Y, Liang M. An adaptive SK technique and its application for fault detection of rolling 
element bearings [J]. Mech. Syst. Signal Process. , 2011, 25(5): 1750-1764.

[3] Zhang S, Wang Y, He S, et al. Bearing fault diagnosis based on variational mode decomposition and total variation denoising[J]. Meas. Sci. Technol., 2016, 27(7): 075101.

[4] Wei Z, Wang Y, He S, et al. A novel intelligent method for bearing fault diagnosis based on affinity propagation clustering and adaptive feature selection [J]. Know.-based Syst., 2017, 116: 1-12.

[5] Lei Y, Lin J, He Z, et al. Application of an improved kurtogram method for fault diagnosis of rolling element bearings [J]. Mech. Syst. Signal Process. , 2011, 25(5): 1738-1749.

[6] Wang Y, He S and Zhang S. Bearing Fault Feature Detecting Based on Nonlocal Means Denoising. The 30th International Congress on Condition Monitoring and Diagnositic Engineering Management Jul. 10-13, 2017, Preston, UK.

[7] Buades A, Coll B, Morel J M. A review of image denoising algorithms, with a new one [J]. Multiscale Model Simul, 2005, 4(2): 490-530.

[8] Wang Y, Markert R, Xiang J, et al. Research on variational mode decomposition and its application in detecting rub-impact fault of the rotor system[J] . Mech. Syst. Signal Process. , 2015, 60: 243-251.

[9] Zhang Y, Zhou G, Zhao Q, et al. Fast nonnegative tensor factorization based on accelerated proximal gradient and low-rank approximation [J]. Neurocomputing, 2016, 198: 148-154.

[10] Cichocki A, Mandic D, De Lathauwer L, et al. Tensor decompositions for signal processing applications: From two-way to multiway component analysis[J]. IEEE Signal Proc. Mag., 2015, 32(2): 145-163.

[11] Fu Y, Dong W. 3D magnetic resonance image denoising using low-rank tensor approximation [J]. Neurocomputing, 2016, 195: 30-39.

[12] Vasilescu M A O, Terzopoulos D. Multilinear Analysis of Image Ensembles: TensorFaces [C] ECCV. Springer-Verlag, 2002:447-460.

[13] Tucker L R. Some mathematical notes on three-mode factor analysis [J]. Psychometrika, 1966, 31(3): 279-311.

[14] Leardi R. Multi - way analysis with applications in the chemical sciences, age smilde, Rasmus Bro and Paul Geladi, Wiley, Chichester, 2004, ISBN 0 - 471 - 98691 - 7, 381 pp[J]. J Chemometr, 2005, 19(2):119-120.

[15] Figueiredo M, Ribeiro B, de Almeida A. Analysis of trends in seasonal electrical energy consumption via non-negative tensor factorization [J]. Neurocomputing, 2015, 170: 318-327.

[16] Muti D, Bourennane S, Marot J. Lower-rank tensor approximation and multiway filtering [J]. SIAM J Matrix Anal. Applicat., 2008, 30(3): 1172-1204.

[17] Muti D, Bourennane S. Multiway filtering based on fourth-order cumulants [J]. EURASIP J Adv. Sig. Pr., 2005, 2005(7): 708571.

[18] Muti D, Bourennane S. Multidimensional estimation based on a tensor decomposition [M]. 2003.

[19] Kolda T G, Bader B W. Tensor decompositions and applications [J]. SIAM Rev., 2009, 51(3): 455-500. 
